\title{
ECONOMÍA EXPERIMENTAL, ECONOMÍA DEL COMPORTAMIENTO Y EL FUTURO DE LA CIENCIA ECONÓMICA COMO DISCIPLINA CIENTÍFICA
}

\author{
MIGUEL A. ALONSO NEIRA*
}

\section{INTRODUCCIÓN}

Hasta recientemente, la economía se observaba como una ciencia no experimental que tenía que basarse en la observación del mundo real en lugar de en experimentos de laboratorio controlados. No obstante, muchos economistas han venido encontrando muy restrictivo el supuesto comúnmente utilizado del homo oeconomicus, motivado por el interés personal y capaz de adoptar decisiones racionales. Por este motivo, en los últimos años la investigación ha adoptado nuevas direcciones.

En la actualidad se está dedicando un gran esfuerzo científico a la contrastación empírica y la modificación de los postulados sobre los que tradicionalmente se ha apoyado la economía como disciplina, especialmente aquellos referidos a los supuestos de racionalidad no limitada, de comportamiento egoísta (o hedonista) ${ }^{1}$, o de absoluto autocontrol. Esta investigación reciente tiene sus raíces en dos tradiciones diferentes pero al mismo tiempo convergentes: por un lado los estudios teóricos y empíricos sobre el proceso de toma de decisiones del ser humano dentro

* Profesor del Departamento de Economía de la Universidad Rey Juan Carlos de Madrid. Doctor en Economía (URJC) y MSc in Economics (Universidad de Essex, Gran Bretaña).

${ }^{1}$ Es decir, el individuo sólo busca la maximización de su propia utilidad. 
de la psicología cognitiva, y por otro lado la contrastación de las predicciones de la teoría económica a través de experimentos de laboratorio - la economía experimental. Actualmente, ambos enfoques se encuentran entre los campos de investigación económica más activos, como puede observarse por la gran cantidad de artículos publicados en los últimos años en las principales revistas de economía. Dos de los últimos premios Nobel, Daniel Kahneman y Vernon Smith, son pioneros en el desarrollo de estos nuevos enfoques.

Kahneman ha recibido el premio Nobel "por haber integrado los logros de la investigación psicológica en el análisis económico, especialmente en lo que se refiere al juicio humano y al proceso de toma de decisiones en estados de incertidumbre». Por otro lado, Smith ha obtenido este galardón «por haber establecido los experimentos de laboratorio como instrumento empírico del análisis económico, especialmente en el estudio de los mecanismos de mercado alternativos».

La contribución más importante de Kahneman se centra en la idea de que el proceso de toma de decisiones del ser humano en momentos de incertidumbre, se desvía de forma sistemática de las predicciones del paradigma racionalista que caracteriza a la economía convencional. Si estas desviaciones de la racionalidad y del comportamiento auto-interesado fuesen pequeñas y idiosincrásicas ${ }^{2}$, podrían en promedio cancelarse, por lo que la teoría económica no estaría demasiado lejos de la verdad cuando predice los resultados a partir de grandes agregados de individuos. Por el contrario, si las desviaciones de la racionalidad y del comportamiento auto-interesado fuesen sistemáticas, sería necesaria una revisión de los propios postulados de la teoría económica.

2 Es decir, ligadas a la personalidad y a la características personales de cada agente. 
Aunque, inevitablemente, a partir de ahora la profesión económica tenderá a agrupar a los dos premios Nobel bajo una misma caracterización (alternativa) del enfoque económico, estos autores despliegan sus experimentos partiendo de diferentes perspectivas y concepciones. Kahneman es un psicólogo y está interesado en cómo trabaja nuestro cerebro y cómo adoptamos nuestras decisiones. Por el contrario, Smith es un economista y está interesado en cómo funcionan los mercados.

Por tanto, parece lógico pensar que con dos métodos y dos objetivos diferentes, estos autores alcancen conclusiones en ocasiones contradictorias. Por ejemplo, el profesor Smith desarrolla su investigación para demostrar lo bien que responde la teoría económica tradicional a los experimentos de laboratorio. Por el contrario, el profesor Kahneman muestra los errores de predicción que comete la teoría económica tradicional, al basarse en los supuestos de racionalidad y de comportamiento auto-interesado, o al apoyarse en la hipótesis de la utilidad esperada. Sus experimentos demuestran que, en un entorno de incertidumbre, el ser humano puede tomar decisiones partiendo de patrones de comportamiento aparentemente irracionales.

En la actualidad, los métodos de la economía experimental y la economía del comportamiento han llegado a convertirse en dos elementos clave de la investigación desarrollada por los estudiantes de postgrado de algunas de las mejores universidades del planeta. Además, en los últimos años, varias universidades de Estados Unidos, Europa, Israel y Japón han abierto centros de investigación dedicados al desarrollo de estos enfoques alternativos al de la economía convencional. 


\section{LA ECONOMÍA EXPERIMENTAL}

Tradicionalmente, la economía se ha caracterizado como una ciencia no experimental que debía descansar en la observación de datos y acciones reales. Muchos economistas observaron que esta concepción era un obstáculo al desarrollo de la economía como una disciplina científica. Únicamente sobre la base de los datos de campo, es difícil decidir si y cuándo una teoría falla, y señalar los aspectos responsables de ese error. El canal de retroalimentación entre la teoría y la observación bajo circunstancias controladas — cuando los nuevos hallazgos resultantes de la experimentación sugieren nuevas teorías y las nuevas teorías sugieren nuevos experimentos- parecía ser algo que no estaba al alcance de la Economía. No obstante, en los últimos años, la proliferación de un creciente número de trabajos basados en la economía experimental ha cambiado radicalmente esta visión. Bajo condiciones de laboratorio controladas, los experimentalitas estudian el comportamiento humano en situaciones que imitan aquellas encontradas en los mercados y en otras formas de interacción económica. La medida en la que los resultados derivados de estos experimentos pueda generalizarse a situaciones de mercado, es algo que todavía permanece bajo un intenso debate.

La investigación experimental en economía tuvo su origen en los trabajos de Chamberlin (1948), quien intentó contrastar la teoría neoclásica de la competencia perfecta por medio de experimentos, y del premio Nobel de Economía Reinhard Selten, que desarrolló los primeros estudios experimentales sobre la formación de los precios en los mercados oligopolísticos. Igualmente, también fueron importantes los trabajos sobre el poder predictivo de la teoría de juegos en un contexto experimental desarrollados por el Nobel de Economía John Nash. No obstante, sin duda alguna el principal investigador en el campo de la economía experimental ha sido el recientemente galardonado Vernon Smith. 
El trabajo más significativo de Smith se refiere a los mecanismos de mercado. Su primer artículo fue inspirado por los experimentos realizados en clase por Chamberlin en 1948. Chamberlin, que fue profesor de Smith en la universidad de Harvard, involucraba a sus alumnos en un proceso de negociación en el que actuaban como compradores y vendedores de un bien ficticio. Este autor, observó los resultados de su experimento como una falsación del modelo neoclásico tradicional de un mercado que opera bajo condiciones de competencia perfecta.

Smith comprobó que los resultados de Chamberlin serían más robustos si los participantes del experimento eran situados en un contexto más parecido al de un mercado real. De este modo, desarrolló una prueba en la que los individuos se dividían en grupos de vendedores y compradores potenciales de una determinada mercancía. Los agentes recibían aleatoriamente los papeles de vendedor y comprador, y a cada vendedor se le asignaba una unidad de mercancía (destinada a la venta), así como un precio de reserva para esa unidad (es decir, un precio mínimo de venta). El precio de reserva del vendedor era una información de la que sólo él disponía. Igualmente, se asignaba a cada comprador un precio de reserva - el precio más alto al que estaría dispuesto a comprar una unidad del bien en cuestión- que también era información privada. Sorprendentemente, Smith encontró que los precios efectivos a los que se intercambiaban las mercancías estaban muy próximos al precio de equilibrio teórico, apoyando de este modo la teoría que pretendía rechazar mediante el desarrollo de este experimento. ${ }^{3}$

Smith ha sido el primer economista en utilizar el laboratorio como un «túnel de viento» para estudiar la ejecución de mecanis-

3 En otras palabras, Smith demostró - contrariamente a lo que él esperabaque cuando los agentes tienen una idea clara de sus propios costes y posibles beneficios, y aunque desconozcan los costes y los beneficios de los demás, tienden a alcanzar un intercambio que responde de forma bastante precisa a las predicciones de la teoría económica convencional. 
mos institucionales como la desregulación, la privatización, y la provisión de bienes públicos. El reciente premio Nobel sostiene que estos mecanismos son normalmente tan complejos que la teoría económica existente no ofrece predicciones precisas, por lo que el método económico experimental se convierte en un procedimiento particularmente útil.

El método experimental de Smith se desvía del enfoque experimental utilizado en el campo de la psicología. ${ }^{4}$ En este sentido, deben destacarse las diferencias que existen entre los métodos experimentales y los métodos fundamentados en la psicología. Mientras los psicólogos han estado esencialmente interesados en el comportamiento individual, Smith desarrolló sus experimentos iniciales con el objeto de analizar los resultados derivados del funcionamiento de los mercados. No obstante, no debe olvidarse que ambos enfoques, al margen de sus diferencias, reflejan dos perspectivas alternativas para entender el comportamiento del ser humano.

\section{LOS FUNDAMENTOS \\ DE LA ECONOMÍA DEL COMPORTAMIENTO}

Hace casi medio siglo, Edwards (1954) introdujo la toma de decisiones como materia de investigación para los psicólogos, definiendo una agenda para los trabajos futuros. Igualmente, Simon (1956) propuso un enfoque para el procesamiento de información y la toma de decisiones basado en el concepto de racionalidad limitada. Sin embargo, la investigación en el ámbito de la psicología cognitiva no llegó hasta los primeros trabajos

${ }^{4}$ Así, este autor destaca la importancia de ofrecer a los individuos los incentivos monetarios adecuados, con el objeto de compensar los efectos distorsionantes de los costes de decisión. También subraya la importancia de diseñar experimentos que se materialicen en pruebas repetidas, de forma que los individuos se familiaricen con la situación experimental y la comprendan. 
de Daniel Kahneman y Amos Tversky. Muchos de los trabajos del primero han sido publicados en las revistas de economía más prestigiosas. Sin duda alguna, este autor ha realizado un trabajo colosal al tender puentes de unión entre la investigación en Economía y la Psicología.

Kahneman y otros autores, han puesto en duda el supuesto de racionalidad económica, al menos en situaciones de decisión complejas o bajo incertidumbre. Varios estudios han descubierto un volumen importante de desviaciones respecto al modelo tradicional de comportamiento económico racional. Por ejemplo, en el mundo real, los individuos no siempre toman sus decisiones evaluando los resultados inciertos según las leyes de la probabilidad (von Neumann y Morgenstern, 1944). En algunas ocasiones, los agentes toman decisiones que violan los principios de maximización de la utilidad esperada.

Kahneman y Tversky descubrieron cómo en situaciones de incertidumbre, los juicios de los individuos se desvían sistemáticamente del tipo de racionalidad propuesto en la teoría económica tradicional. Estos autores contemplaron que, frecuentemente, los individuos son incapaces de analizar plenamente situaciones que implican juicios económicos y probabilísticos. En esas situaciones, el ser humano se apoya en ciertos atajos heurísticos (modelos mentales previos, emociones y actitudes, la memoria de situaciones y decisiones pasadas...) que, en ocasiones, están sistemáticamente sesgados.

Kahneman concluye que la evidencia existente sobre los juicios del ser humano, demuestra que el razonamiento de las personas viola las leyes básicas de la probabilidad de una forma sistemática. En este sentido, este autor cuestiona la validez empírica de uno de los fundamentos de la teoría económica tradicional: la evidencia disponible, no sólo indica que los juicios sino también la toma de decisiones bajo incertidumbre, se desvían de una forma sistemática del enfoque económico tradicional, fundamentado en la hipótesis de la utilidad esperada. 
En 1988, Maurice Allais planteó por primera vez la existencia de desviaciones respecto a esta hipótesis inicialmente desarrollada por von Neumann-Morgenstern. Estas desviaciones pasaron a constituir la conocida «Paradoja de Allais». En los últimos años, Kahneman ha proporcionado evidencia de la existencia de estas desviaciones, ofreciendo una teoría alternativa a la de la hipótesis de la utilidad esperada: la "prospect theory», una fórmula de análisis según la cual la gente concede una mayor importancia a una ganancia segura que a una probable, aunque se prevea que esta última pueda ser superior.

\section{CONCLUSIÓN}

Con el desarrollo de la economía fundamentada en la psicología y la economía experimental, Daniel Kahneman y Vernon Smith se han desviado considerablemente del enfoque económico tradicional apoyado en el supuesto de racionalidad perfecta. La investigación en economía tiende cada vez más a tener en cuenta que la racionalidad humana es limitada, y que las preferencias de la gente son interdependientes y no puramente individualistas (véase al respecto Brandts, 2002).

Daniel Kahneman ha utilizado las ideas contenidas en la psicología cognitiva respecto a los procesos mentales para responder a preguntas, formar juicios, y hacer elecciones, con el objeto de ayudarnos a mejorar nuestra comprensión de cómo la gente adopta sus decisiones económicas. En este sentido, se ha convertido en el investigador más influyente en el campo de la economía del comportamiento. Su influencia se extiende a otras disciplinas como las ciencias naturales, las humanidades y la medicina.

Vernon Smith es el economista más influyente en el lanzamiento de los experimentos de laboratorio como un método empírico en el desarrollo de la Economía como una ciencia. Al contrario que Kahneman, no comienza desafiando la teoría 
económica tradicional en lo que se refiere al proceso de toma de decisiones racionales. En su lugar, contrasta hipótesis alternativas respecto a la ejecución de los mercados.

Mientras que Kahneman centra sus experimentos en la toma de decisiones de los agentes individuales, Smith tiene como principal objetivo la interacción entre los agentes en entornos de mercado específicos, habiéndose convertido en el máximo exponente de la economía experimental.

En los últimos años, se ha observado un creciente desarrollo de la investigación que combina la psicología y la economía experimental. La evidencia experimental indica que ciertos fenómenos psicológicos - como la racionalidad limitada o el comportamiento hedonista (búsqueda de la máxima utilidad individual) - son factores importantes que subyacen a una amplia variedad de resultados económicos. Este razonamiento podría llevar a la sustitución de algunos elementos que tradicionalmente han caracterizado a la teoría económica convencional. En ese sentido, el trabajo de los dos últimos premios Nobel podría suponer una auténtica revolución en la investigación económica de los próximos años. El transcurso del tiempo nos dirá si esto realmente llega a producirse. Mientras tanto, permítanme mostrar mis reservas respecto a que esta posibilidad llegue a ser real.

Por un lado, aunque Smith se alinea con los austriacos al admitir la importancia de la observación de la acción humana en una economía de mercado, también es cierto que viola una premisa central de la metodología austríaca: las leyes de la acción humana no pueden contrastarse a través de la falsación. Por tanto, la economía experimental no puede probar que las leyes de la economía sean ciertas.

Por otro lado, tal y como sostiene Shostak (2002), los hechos pertenecientes a la acción humana no pueden aislarse y dividirse en elementos simples. Las realidades relativas a la acción humana son hechos históricos complejos que han surgido como consecuencia de muchos factores causales. 
Finalmente, los trabajos de Kahneman llevan a la conclusión de que la gente no siempre se comporta de forma racional, es decir según las premisas de la economía convencional. Sin embargo, lo que Kahneman realmente ha descubierto no tiene nada que ver con el hecho de que la gente sea racional no, sino con el postulado erróneo de que las preferencias son y se mantienen constantes. Esto es sinónimo de decir que los individuos son como máquinas que nunca cambian sus mentes. Obviamente las preferencias pueden cambiar, y lo que Kahneman ha descubierto es que el comportamiento de los agentes en una economía se desvía de la caracterización, más o menos artificial, del homo oeconomicus. No obstante, permítanme que trate todos estos aspectos en un futuro trabajo. 


\section{BIBLIOGRAFÍA CITADA}

Altman, D. (2002). «A Nobel that bridges Economics and Psychology». The New York Times (nytimes.com).

http:/ / nytimes.com/2002/10/10/business/10PRIZ.html

ANDERSON, W. L. (2002). «Two Cheers for the 2002 Nobel Laureates».

Ludwig von Mises Institute.

http://www.mises.org/fullstory.asp?control=1067

BRANDTS, J. (2002) «Experimentos con la economía». 5Días.com.

http: / / . / / articulo.html?anchor $=$ cd spor $\&$ xref $=20021011$

cdscdiopi_5\&type $=$ Tes\&date $=2002101$

CHAMBERLiN, E. H. (1948). «An experimental imperfect market». Journal of Political Economy, $\mathrm{n}^{\circ}$ 56: 95-108.

EDWARDS, W. (1954). «Behavioral decision theory». Annual Review of Psychology, $\mathrm{n}^{\circ}$ 12: 473-498.

SHOSTAK, F. (2002). «Behavioral, Experimental, and Austrian Economics». Ludwig von Mises Institute.

http://www.mises.org/fullstory.asp?control=1082

SIMON, H. A. (1956). "Rational choice and the structure of the environment». Psychological Review, $\mathrm{n}^{\circ}$ 63: 129-138.

THE ROYAL SWEDISH ACADEMY OF SCIENCES (2002). «Foundations of Behavioral and Experimental Economics: Daniel Kahneman y Vernon Smith». Advanced Information on the Prize in Economic Sciences 2002. http:/ / www.kva.se 